\title{
Metabolite Profiling and Anti-Aging Activity of Rice Koji Fermented with Aspergillus oryzae and Aspergillus cristatus: A Comparative Study
}

\author{
Hyunji Lee ${ }^{1,+}$, Sunmin Lee ${ }^{1,+}$, Seoyeon Kyung ${ }^{2}$, Jeoungjin Ryu ${ }^{2} \oplus$, Seunghyun Kang ${ }^{2}$, Myeongsam Park ${ }^{2}$ \\ and Choonghwan Lee ${ }^{1,3, *}$ \\ 1 Department of Bioscience and Biotechnology, Konkuk University, Seoul 05029, Korea; \\ oasis7184@naver.com (H.L.); duly123@naver.com (S.L.) \\ 2 COSMAX BTI R\&I Center, Pangyo inno Valley E, 255 Pangyo-ro, Bundang-gu, Seongnam-si 13486, Korea; \\ sykyung@cosmax.com (S.K.); jjyou@cosmax.com (J.R.); shyunk@cosmax.com (S.K.); \\ msampark@cosmax.com (M.P.) \\ 3 Research Institute for Bioactive-Metabolome Network, Konkuk University, Seoul 05029, Korea \\ * Correspondence: chlee123@konkuk.ac.kr; Tel.: +82-2-2049-6177 \\ + Both authors contributed equally to this work.
}

check for updates

Citation: Lee, H.; Lee, S.; Kyung, S.; Ryu, J.; Kang, S.; Park, M.; Lee, C.

Metabolite Profiling and Anti-Aging Activity of Rice Koji Fermented with Aspergillus oryzae and Aspergillus cristatus: A Comparative Study. Metabolites 2021, 11, 524. https:// doi.org/10.3390/metabo11080524

Academic Editor: Thusitha

W. Rupasinghe

Received: 13 July 2021

Accepted: 4 August 2021

Published: 8 August 2021

Publisher's Note: MDPI stays neutral with regard to jurisdictional claims in published maps and institutional affiliations.

Copyright: (c) 2021 by the authors. Licensee MDPI, Basel, Switzerland. This article is an open access article distributed under the terms and conditions of the Creative Commons Attribution (CC BY) license (https:// creativecommons.org/licenses/by/ $4.0 /)$

\begin{abstract}
Rice koji, used as a starter for maximizing fermentation benefits, produces versatile end products depending on the inoculum microbes used. Here, we performed metabolite profiling to compare rice koji fermented with two important filamentous fungus, Aspergillus oryzae and A. cristatus, during 8 days. The multivariate analyses showed distinct patterns of primary and secondary metabolites in the two kojis. The rice koji fermented with $A$. oryzae (RAO) showed increased $\alpha$ glucosidase activity and higher contents of sugar derivatives than the one fermented with $A$. cristatus (RAC). RAC showed enhanced $\beta$-glucosidase activity and increased contents of flavonoids and lysophospholipids, compared to RAO. Overall, at the final fermentation stage (8 days), the antioxidant activities and anti-aging effects were higher in RAC than in RAO, corresponding to the increased metabolites such as flavonoids and auroglaucin derivatives in RAC. This comparative metabolomic approach can be applied in production optimization and quality control analyses of koji products.
\end{abstract}

Keywords: rice koji; microbe; solid-state fermentation; anti-aging effect; antioxidant activity

\section{Introduction}

Fermentation, which has a history of thousands of years, is increasingly being recognized as a method of enhancing nutrition and bioactivities of food products, in addition to processing and preserving them [1]. Rice koji is made by solid-state fermentation using steamed rice grains inoculated with microorganisms to secrete enzymes and produce beneficial metabolites. In recent years, various attempts to create delicate fermentation conditions have led to advanced fermentation efficacy and better food palatability [2,3]. Due to its advantages rice koji finds applications in industrial fields such as fermented foods and beverages and cosmetics [4-6].

Reactive oxygen species (ROS) are generated under conditions of oxidative stress and are by-products of aerobic metabolism. These free radicals can induce the degradation of biomolecules, resulting in oxidative damages, such as inflammation and acceleration of the skin aging process [7]. To develop a balance between ROS production and elimination, ROS scavengers, known as antioxidants, play an important role in alleviating oxidative stress and are mainly obtained from natural sources [8]. These free radicals are involved in the aging process, and scavenging them through the intake of antioxidants from natural sources is crucial in delaying aging [9]. In recent years, many studies have reported that rice koji can enhance the potential antioxidant activities of raw materials by improvement of the fermentation substrate $[10,11]$. 
The skin's extracellular matrix (ECM) consists of collagen and elastin fibers, which promote elasticity of the skin to restore and maintain its original shape and state [12]. The destruction of the dermal ECM is an indicator of aging. It occurs due to the upregulation of collagen-degrading matrix metalloproteinase-1 (MMP-1), also known as collagenase. Therefore, studies of various phytochemicals that can slow down the skin aging process by stimulating collagen and elastin synthesis and inhibiting MMP-1 are increasing [13-16]. Seo et al. showed that fermented rice bran affects skin fibroblast collagen, inflammatory factor (IL-a), and MMP-1 [17]. Hence, various compounds found in rice, such as flavonoids and phenolic acids have antioxidant activity, and fermented rice koji has the potential to ameliorate the skin photoaging by UV radiation [18].

Aspergillus, a filamentous fungus, is a typical inoculum microbe for producing many beneficial metabolites such as simple sugars, fatty acids, and amino acids from koji in Asia. In particular, Aspergillus oryzae is the most common microorganism used in the production of koji because of its ensured safety and various enzymes, such as amylase, protease, and peptidase [19]. Aspergillus cristatus is used in tea fermentation, such as Fuzhuan brick tea, which has probiotics and protects against UVB-induced photoaging [20,21]. It has also been reported to enhance the antioxidant activity of various other raw materials [22,23]. Currently, efforts are increasingly being dedicated to improve the quality of fermentation starters $[4,24]$. Previous study have shown a comparative metabolic study of Aspergillus and Bacillus, widely used in rice koji [25]. However, there is a scarcity of information about the metabolomic differences between the same genera but different species of fungi. To select optimal microbes that can be introduced in the market for health with nutraceutical and cosmeceutical applications, there is a need for a comprehensive understanding of the metabolism of different inoculum microbes by comparing their bioactivity and metabolites.

In this study, we profiled the metabolites of rice koji fermented with different Aspergillus spp. (A. cristatus and A. oryzae) in terms of metabolomics to compare the metabolism of the two filamentous fungi. We also measured enzyme activity, antioxidant activity, and RNA expression of skin anti-aging factors (collagen, elastin, and MMP-1) to compare the two koji. Furthermore, we conducted correlation analysis to suggest potential candidate metabolites that contribute to antioxidant activity and skin anti-aging effects. A comprehensive analysis of MS-based metabolite profiling for comparing the two koji inoculums established a relationship between enzyme activities, metabolomes, and bioactivities. Here, we present a blueprint of the overall metabolic state, correlated with the bioactivities of the two different koji inoculums.

\section{Results}

\subsection{Metabolic Profiling for Rice Koji Fermented with Different Aspergillus spp.}

Different metabolomes of rice koji samples inoculated with A. cristatus or A. oryzae were compared using multivariate analysis according to the GC-MS and LC-MS data set. The principal component analysis (PCA) score plot obtained from the UHPLC-LTQ-OrbitrapMS/MS and GC-TOF-MS revealed a total variance of 40.9\% (PC1, 22.01\%; PC2, 18.89\%) and $52.88 \%$ (PC1, 34.70\%; PC2, 18.18\%), respectively (Figure 1A,B). Both PCA results indicated that the starting point of the fermentation was assembled, but consequently distinguished by different inoculation fungi according to different fermentation times. Partial least squares discriminant analysis (PLS-DA) elucidated statistical patterns same to the metabolite distribution in PCA (Supplementary Figure S1A,B).

As shown in the PCA obtained from the UHPLC-LTQ-Orbitrap-MS/MS analyses (Figure 1A), there are significant differences in the eighth day, and both eight day samples were subjected to an orthogonal partial least square discriminant analysis (OPLS-DA), which showed a clear separation by OPLS component 1 , accounting for $86.11 \%$ of the variance in data (Supplementary Figure S1C). The 31 metabolites were selected from UHPLC-LTQ-Orbitrap-MS/MS data, which is considered a major contributor to the discrepancy in eighth day rice kojis fermented with two different inoculum microbes based on their variable importance in projection values (VIP $>1.0)$ and $p$-values $(p<0.05)$ from 
OPLS-DA analysis (Supplementary Table S1). These metabolites included 2 carboxylic acids, 5 phenolic acids, 7 flavonoids, 2 long chain fatty acids, 11 lysophospholipids, and 4 hydroquinones. The metabolites were tentatively identified by comparing published literature (molecular weight, molecular formula, retention time, mass fragment patterns, and UV absorbances) and data from an in-house library.

(A)

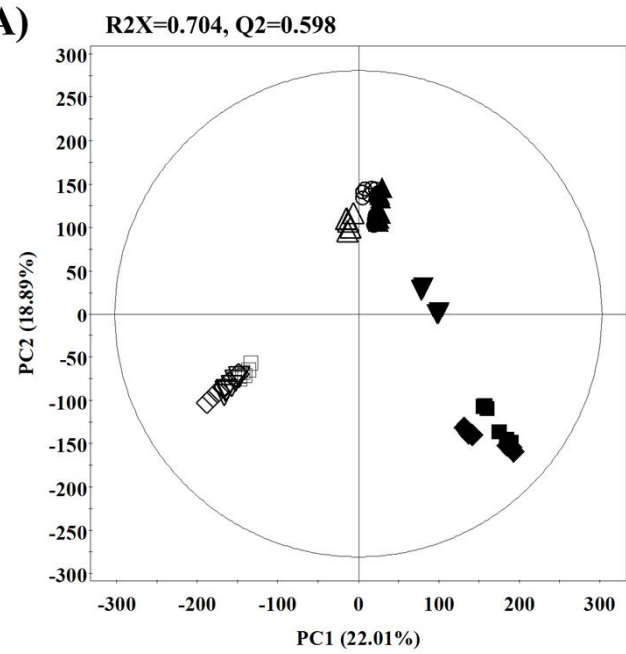

(B)

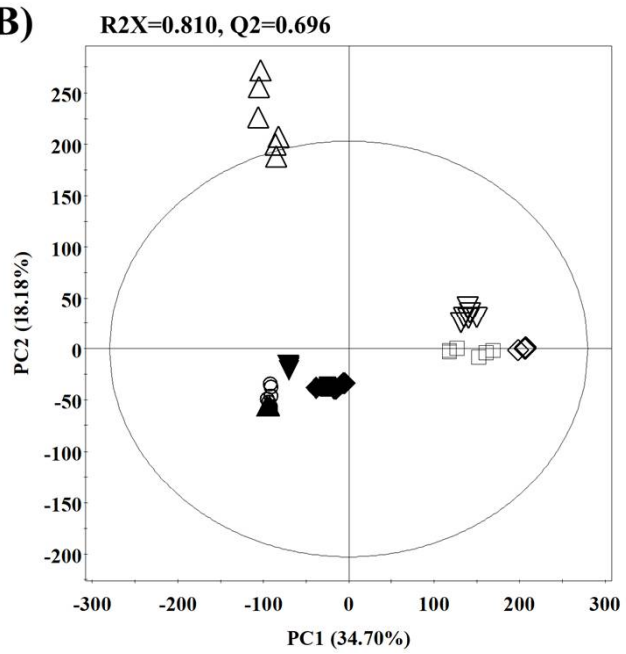

Figure 1. Principal component analysis (PCA) score plot from the (A) UHPLC-LTQ-Orbitrap-MS/MS and (B) GC-TOF-MS data sets of rice koji fermented with Aspergillus cristatus or A. oryzae. (filled symbols, A. cristatus; unfilled symbols, A. oryzae; $\bullet, \bigcirc, 0$ day; $\mathbf{\Lambda}, \triangle, 2$ day; $\mathbf{\nabla}, \nabla, 4$ day; $\mathbf{\square}, \square, 6$ day; $\diamond, 8$ day).

In addition, 20 primary metabolites from the GC-TOF-MS data that are contributed to distinctions in rice koji (VIP $>1.0$ and $p<0.05$ ) were determined by GC-TOF-MS to contain four organic acids, twelve sugar derivatives, and four fatty acids (Supplementary Table S2). These metabolites were identified by using standard compounds for comparing mass fragment patterns and retention times, in addition to an in-house library.

2.1.1. Temporal Metabolomes for Rice Koji with Different Aspergillus spp. Inoculation According to Fermentation Time

The metabolic pathways of rice koji dependent on different inoculation microbes were represented by a heat map to visualize metabolite change patterns in accordance with fermentation times (Figure 2). The color on a blue-to-red gradient represents the meannormalized relative abundance of each metabolite under each experimental condition. The trends of most metabolites in rice koji fermented with A. cristatus (RAC) and A. oryzae (RAO) showed a gradually increasing pattern with fermentation time. The metabolites associated with carbohydrate metabolism mostly represented an increasing pattern except for glucose, xylose, sucrose, and maltose, which are sugars. In addition, phenolic acid, flavonoids, and hydroquinone contents were enhanced with fermentation time, except for ferulic acid. Among the fatty acids, most metabolites showed an increasing pattern, while pinellic acid showed a decrease. Lysophospholipids presented disparate patterns with different fermentation times and inoculation fungi. 


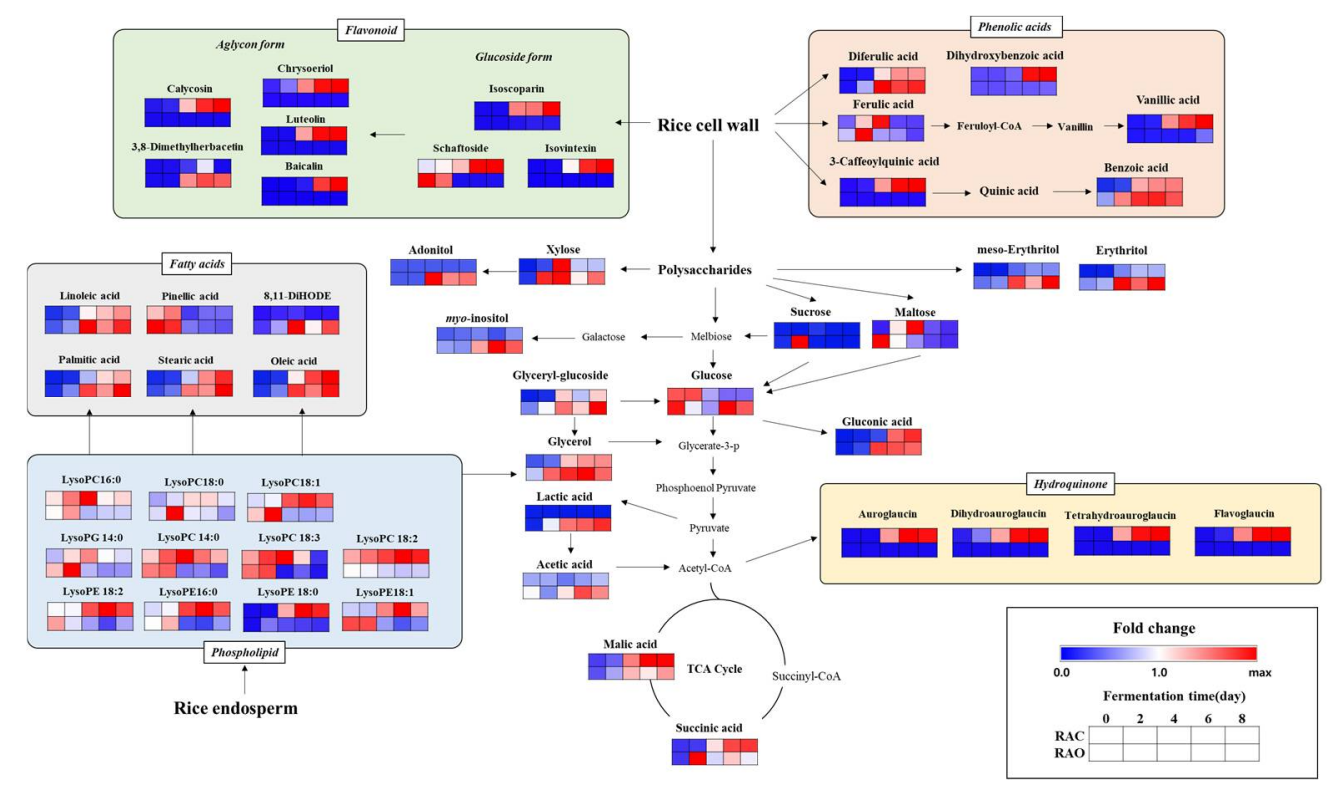

Figure 2. Scheme of the metabolic pathway and relative levels of metabolites in rice koji fermented with Aspergillus cristatus or A. oryzae. The pathway was adapted from the Kyoto Encyclopedia of Genes and Genomes (KEGG) database and modified. The colored squares represent the fold changes (blue to red) normalized by the average of all values for each metabolite.

2.1.2. Relative Disparity in the Level of Discriminant Metabolites in Rice Koji Fermented by A. cristatus or A. oryzae

As shown in Figure 2, the contents of primary and secondary metabolites exhibited different patterns in accordance with different inoculation fungi. In the case of glucose, which is the center of carbohydrate metabolism, the patterns of $A$. cristatus koji showed a decrease, whereas $A$. oryzae koji showed decreasing patterns at the initial fermentation point but gradually increased until the final fermentation point. Furthermore, the sugar alcohols were higher in RAO than in RAC. Particularly, auroglaucin derivatives were enhanced significantly only in RAC because they are a unique pigment compound produced by A. cristatus. In addition, most flavonoids were increased significantly in RAC compared to RAO, except for 3,8-dimethylherbacetin. Among the phenolic acids, diferulic acid and benzoic acid were increased in both samples, but dihydroxybenzoic acid, caffeoquinic acid, and vanillic acid were increased only in RAC. Lysophospholipids increased in RAC, but a contrasting tendency was observed in RAO. Fatty acids showed greater patterns of increase in RAO than in RAC.

\subsection{Comparison of Enzymatic Production and Bioactivity in Rice Koji Fermented with Different Microorganisms}

To compare the phenotypes of RAC and RAO, we evaluated the enzyme activity and anti-aging effects on skin cells, and antioxidant activity, total flavonoid contents (TFC) and total phenolic contents (TPC) (Figure 3). Enzyme production of both the kojis increased with fermentation time, except for $\alpha$-amylase in RAO. Interestingly, the $\alpha$-glucosidase content was twice higher in RAO than in RAC with 10.12 and 3.52 units respectively; in contrast, $\beta$-glucosidase content was forth higher in RAC than in RAO with 19.05 unit and 5.49 unit respectively, in accordance with fermentation times. The functional phenotype of both the kojis (antioxidant activity and skin anti-aging factor) indicated that the rice koji with $A$. cristatus had higher antioxidant activities in ABTS, DPPH, FRAP at the final fermentation time (8 days) with 1.05, 0.40, 0.66 TEAC (Trolox equivalent antioxidant capacity) respectively. Additionally, the content of flavonoid was higher in RAC than RAO with $0.07 \mathrm{NE}$ (naringin equivalent) and $0.01 \mathrm{NE}$ respectively. Whereas the content of total phenol was higher in RAO than RAC with 0.32 EGA (equivalent gallic acid) and 0.28 EGA respectively. The results of skin anti-aging factor (elastin, collagen, and MMP-1) indicated 
that at the termination of the fermentation, RAC had higher relative collagen and elastin RNA expression level with 7.77 and 13.76 and lower relative MMP-1 RNA expression level with 2.35 compared to $\beta$-actin. Meanwhile, RAO showed a gradual increase in RNA expression of elastin and collagen following fermentation.

(A)
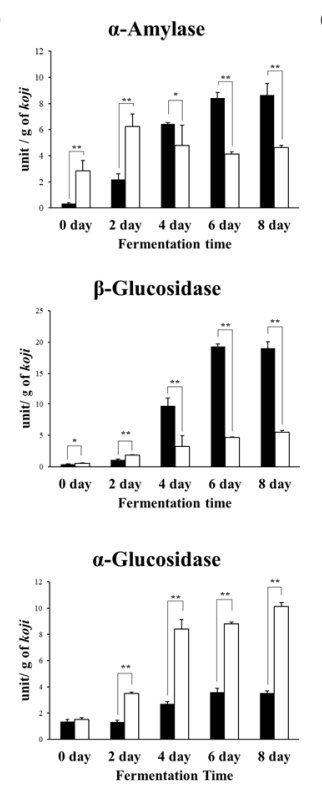

(B)

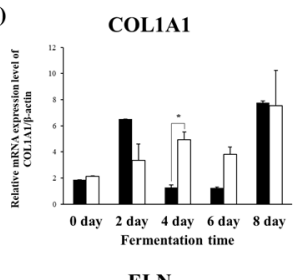

ELN

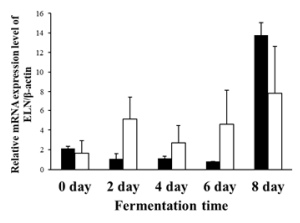

MMP-1

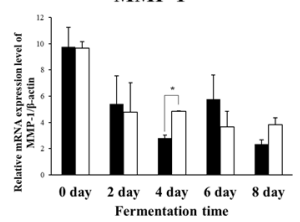

(C)
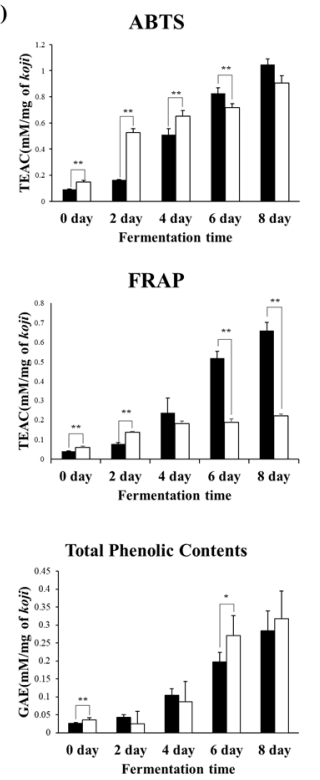
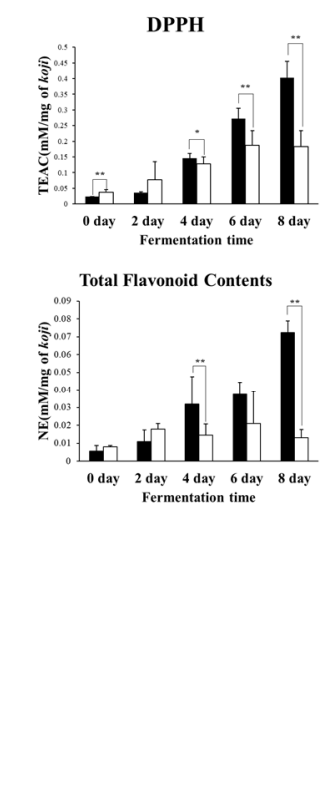

Figure 3. Comparison of enzyme production (A), skin anti-aging factor (B) and antioxidant activity, total flavonoid contents (TFC), total phenolic contents (TPC) (C) in rice koji fermented with different Aspergillus spp. (black color, A. cristatus; white color, $A$. oryzae). The enzymatic activities are $\alpha$-amylase activity, $\beta$-glucosidase activity, and $\alpha$-glucosidase activity (A). The relative mRNA expression level is measured for the following: collagen (COL1A1), elastin (ELN), and matrix metalloproteinase-1 (MMP-1) (B). The antioxidant activities depicted are ABTS, DPPH radical scavenging, FRAP, total flavonoid content, and total phenolic content $(\mathbf{C})$. Significant differences between different inoculation microbes were identified by $t$-test $\left({ }^{*} p<0.05,{ }^{* *} p<0.01\right)$.

To determine the metabolites that potentially contributed to bioactivity, a correlation analysis between fermented koji metabolites and bioactivities was conducted (Supplementary Figure S2). Overall, the Pearson's correlation coefficient map showed the RAC higher correlation with bioactivities than RAO. In RAC, organic acids, flavonoids, lysophospholipids, fatty acids, hydroquinone, sugar derivatives were showed high positive correlation with bioactivities. For RAO, organic acids, flavonoids and fatty acids, and sugar derivatives were indicated positive correlation with bioactivities. The metabolites that had a Pearson's correlation coefficient value higher than 0.5 are represented in a network map (Figure 4). In both the koji products, organic acids, fatty acids, flavonoids, and sugar derivatives were potential contributors of bioactivities. RNA expression of elastin was associated with metabolites of RAC, whereas RNA expression of collagen was associated with metabolites of RAO. In addition, TFC showed a correlation with RAC. Furthermore, lysophospholipids and hydroquinone were strong antioxidant activity contributors to RAC. 


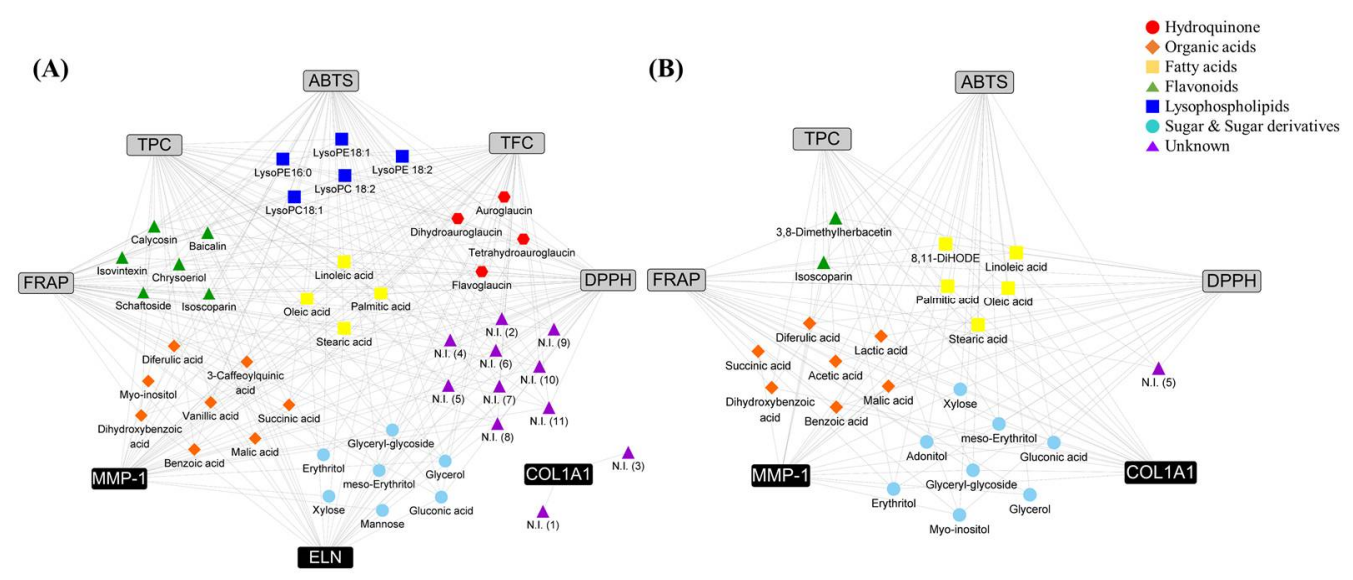

Figure 4. The metabolites that have a Pearson's correlation coefficient value higher than 0.5 are represented by a network map in rice koji fermented with (A) Aspergillus cristatus or (B) A. oryzae. The box symbols represent bioactivities (gray color, antioxidant activity, TPC and TFC; black color, skin anti-aging effect on cell) and the colored symbols indicate the metabolites (same series were distinguished by different color and shape; $\bullet$, hydroquinone; $\bullet$, organic acids; $\mathbf{\square}$, fatty acids;

$\boldsymbol{\Lambda}$, flavonoids; $\mathbf{\square}$, lysophospholipids; $\bullet$, sugar and sugar derivatives; $\boldsymbol{\Lambda}$, unknown).

\section{Discussion}

Different parts of rice, such as husk, bran, embryo, and endosperm, from the surface to the interior, have different chemical compositions [26]. In particular, rice bran contains various phenolic acids and flavonoids, which are known to exhibit antioxidant activity. In addition, the rice cell wall is composed of an arabinoxylan structure that includes xylose, arabinose, ferulic acid, and diferulic acid [27]. The rice cell wall is generally hard to penetrate, and rice koji offers the advantage of easy penetration of the rice cell wall by various enzymes such as protease and glucosidase from inoculum microbes [24]. Therefore, rice koji shows higher levels of tyrosinase inhibitory activity and antioxidant activities than its raw materials because it contains valuable enriched compounds [28].

We followed the metabolomics approach for rice koji fermented with two different filamentous fungi, which elucidated significant differences in enzyme activity, production of metabolites, and bioactivities. Activities of various enzymes such as $\alpha$-amylase, $\alpha$ glucosidase, and $\beta$-glucosidase produced by the inoculated A. cristatus and A. oryzae increased with fermentation time (Figure 3). Because these enzymes break down the arabinoxylan structure, diverse phenolic acids were separated from the rice cell wall in both the samples, as shown in Figure 2. These phenolic acids are potential antioxidants that alleviate oxidative stress [29]. Thus, antioxidant activities and TPC assay increased with increasing fermentation time as the phenolic acid content was enhanced (Figures 2 and 4). In particular, RAC has a higher content of flavonoids than RAO because it has a higher level of $\beta$-glucosidase, which hydrolyzes the $\beta$-glycosidic linkage from the rice cell wall, during the growth of $A$. cristatus in rice koji. In addition to detaching from the rice cell wall, $\beta$-glucosidase hydrolyzes the flavonoid glucoside form to an aglycon form that possesses higher antioxidant activity [30]. The increased flavonoid glucoside form and aglycon form increases antioxidant activities such as ABTS, DPPH, FRAP, and TFC, which may affect the antioxidant activity of RAC, as shown in the correlation network map (Figure 4). This phenomenon was also observed in a previous study that showed biotransformation of glucoside isoflavones to aglycones and increasing patterns in antioxidant activity according to fermentation time in soybean fermented with A. cristatus [31].

RAO has a higher level of $\alpha$-glucosidase activity that cleaves $\alpha$-glycosidic linkages and generates higher content of glucose. Besides the fact that the glucose is the main carbon source for fungus, in RAC, the glucose level decreased following fermentation because it was used for the synthesis of secondary metabolites such as auroglaucin derivatives, which are distinctive pigment compounds produced by A. cristatus, and not A. oryzae. Previous studies have reported that auroglaucin derivatives have activity in DPPH and assumed as 
the potential antioxidant compounds [32]. Furthermore, the collapsed rice cell wall could allow enzymes to penetrate into the innermost parts of the rice [24]. Hence, more and more metabolites could be extracted freely without interruption from the rice outer wall.

In the correlation network map between bioactivities and metabolites of both RAO and RAC (Figure 4), the common tendencies were that flavonoids, organic acids, sugar derivatives, and fatty acids were suggested as potential contributors to bioactivities. Flavonoids and phenolic acids are renowned antioxidants and have many advantages with respect to various functions. Due to their ability to alleviate oxidative stress, they are used to enhance food quality and ameliorate skin aging [33]. Additionally, a previous study has reported that fatty acids and antioxidants could create a synergistic effect for the prevention and management of skin aging [34].

On the other hand, auroglaucin and lysophospholipid derivatives serve as additional contributors to metabolites in RAC [35]. The auroglaucin derivatives have antioxidant activities, as stated above, and therefore, we assume that they have the potential to terminate free radical chain reactions to relieve skin stresses. Yahagi et al. demonstrated that lysophospholipids could maintain skin moisturization by enhancing the expression of factors associated with the skin barrier and hydration functions in the skin [36]. Moisturization is a vital factor for healthy skin because dryness induces skin impairment that is characterized by roughness, scaly skin and fine wrinkles $[37,38]$. We estimate that auroglaucin and lysophospholipids have better skin anti-aging effects at the final fermentation stage in RAC than in RAO. Zhao et al. demonstrated that Fuzhuan brick tea, which contains the dominant fungus $A$. cristatus, can inhibit photoaging via quenching of ROS and triggering of Nrf2 signaling cascades [21]. Therefore, we assume that RAC offers a higher anti-aging potential than RAO by acting through indirect routes such as establishing better skin conditions for abundant moisture and relieving free radical stress.

Overall, we believe that the enhanced fatty acids, phenolic acids, flavonoids, lysophospholipids, and hydroquinones may increase antioxidant activities and improve RNA expression of elastin and collagen, as well as suppress RNA expression of MMP-1, at the end of fermentation. These compounds exhibited different patterns of change in metabolites according to the inoculum fungus and affected various bioactivities. This study elucidated the difference in overall metabolism between different species of the same Aspergillus genus by using a metabolomics approach. In addition, different enzyme activities influenced the production of different metabolites and induced different bioactivities in RAO and RAC.

\section{Materials and Methods}

\subsection{Chemicals and Reagents}

Analytical grade methanol, acetonitrile, and water were purchased from Fisher Scientific (Pittsburgh, PA, USA). Reagent grade chemicals, including methoxyamine hydrochloride, pyridine, and $\mathrm{N}$-methyl- $\mathrm{N}$-(trimethylsilyl)-trifluoroacetamide (MSTFA), were obtained from Sigma Chemical Co. (St. Louis, MO, USA).

Analytical-grade chemicals, namely, acetic acid, 2,2-azinobis(3-ethylbenzothiazoline6-sulfonic acid) diammonium salt (ABTS), Folin-Ciocalteu's phenol, formaldehyde solution, formic acid, methoxyamine hydrochloride, $\mathrm{p}$-nitrophenol, $\mathrm{p}$-nitrophenol $\beta$-Dglucopyranoside (p-NPG), potassium persulfate, pyridine, sodium hydroxide, sodium acetate, starch, N-methyl- $\mathrm{N}$-(trimethylsilyl)trifluoroacetamide (MSTFA), 6-hydroxy-2,5,7,8tetramethylchroman-2-carboxylic acid (Trolox), and tyrosine were purchased from SigmaAldrich (St. Louis, MO, USA). Sodium carbonate, sodium dihydrogen phosphate, and disodium hydrogen phosphate were purchased from Junsei Chemical Co., Ltd. (Tokyo, Japan).

\subsection{Sample Preparation and Extraction}

The koji molds A. oryzae KCCM 11372 (Korean Culture Center of Microorganism, KCCM; Republic of Korea) and A. cristatus (Aspergillus cristatus Cosmax-GF from Cosmax 
BTI R\&I center; Seongnam, Korea) were used for fermentation of rice and separately inoculated. Each microorganism was maintained on malt extract agar (malt extract, $20 \mathrm{~g}$; glucose, $20 \mathrm{~g}$; peptone, $1 \mathrm{~g}$; agar, $20 \mathrm{~g} / \mathrm{L}$ ) at $28^{\circ} \mathrm{C}$. The bioprocess of fermentation steps for koji production was adapted from Lee et al. [11]. The rice koji samples fermented with A. oryzae and A. cristatus were harvested every 2 days (from day 0 to day 8 ) and stored at deep freezing conditions $\left(-80^{\circ} \mathrm{C}\right)$ until further analyses. All samples were prepared with two biological replicates.

The method of extraction of rice koji sample was adapted from Lee et al. with slight modifications [11]. Briefly, the pulverized freeze-dried rice koji samples $(5 \mathrm{~g})$ were extracted by adding $80 \%$ aqueous ethanol $(40 \mathrm{~mL})$ and agitating on an orbital shaker $(200 \mathrm{rpm}$ for $24 \mathrm{~h}$ ) at room temperature. After centrifugation of the samples at $10,000 \mathrm{rpm}$ for $5 \mathrm{~min}$ at $4{ }^{\circ} \mathrm{C}$, the supernatants were filtered with a $0.22 \mu \mathrm{m}$ Millex GP filter (Merck Millipore, Billerica, MA, USA). The filtered sample extracts were dried using a speed vacuum concentrator (Hanil, Seoul, Korea) and the dry weight was measured to evaluate the extraction yield.

\subsection{GC-TOF-MS Analysis}

The derivatization steps of extracted rice koji samples were as described by Lee et al. [11]. GC-TOF-MS analysis was conducted on an Agilent 7890A GC system (Santa Clara, CA, USA) with a Pegasus HT TOF-MS (Leco Corporation, St. Joseph, MI, USA). The carrier gas (helium) was used with an RTx-5MS (30 m length $\times 0.25 \mathrm{~mm}$ inner diameter, J\&W Scientific, Folsom, CA, USA) at a constant flow rate of $1.5 \mathrm{~mL} / \mathrm{min}$. The temperatures of the injector and ion source were maintained at 250 and $230^{\circ} \mathrm{C}$, respectively. The oven temperature was maintained at $75^{\circ} \mathrm{C}$ for $2 \mathrm{~min}$ and then increased to $300^{\circ} \mathrm{C}$ at $15^{\circ} \mathrm{C} / \mathrm{min}$, which was sustained for $3 \mathrm{~min}$. Then, $1 \mu \mathrm{L}$ of sample was injected with a mass scan range of $\mathrm{m} / \mathrm{z}$ 50-800. All sample analyses were performed with three analytical replicates.

\subsection{UHPLC-LTQ-Orbitrap-MS Analysis}

The extracted rice koji samples were analyzed for secondary metabolites using ultrahighperformance liquid chromatography linear trap quadrupole orbitrap tandem mass spectrometry (UHPLC-LTQ-Orbitrap-MS/MS) using the protocols described by Kwon et al. [39]. Each sample was separated using a Phenomenex KINETEX ${ }^{\circledR}$ C18 column $(100 \mathrm{~mm} 2.1 \mathrm{~mm}$, $1.7 \mathrm{~m}$ particle size; Torrance, CA, USA). The mass spectra and photodiode array range in both positive and negative ion modes were tuned for $\mathrm{m} / \mathrm{z} 100-1000$ and $200-600 \mathrm{~nm}$, respectively.

\subsection{Data Processing and Statistical Analysis}

The raw GC-TOF-MS and UHPLC-LTQ-Orbitrap-MS/MS data were transformed to netCDF ( ${ }^{*}$.cdf) format using Leco ChromaTOF and Thermo Xcalibur software, respectively. The respective net CDF ${ }^{*}$.cdf) files were subjected to MetAlign (http:/ / www.metalign.nl (accessed on 13 July 2021)) software-mediated data processing using the protocols described by Lee et al. [11,24]. The mass spectrometric data, which represent the suitable peak mass $(\mathrm{m} / \mathrm{z})$, retention times $(\mathrm{min})$, and peak area information as variables, were evaluated using SIMCA-P+ 12.0 software (Umetrics, Umea, Sweden) for multivariate statistical analysis. Before principal component analysis (PCA), partial least squares discriminant analysis (PLS-DA), and orthogonal partial least square discriminant analysis (OPLS-DA), the data sets were log-transformed, and unit variance was scaled to compare the rice koji fermented with different fungi. PASW Statistics 18 (SPSS, Inc., Chicago, IL, USA) was used to test for significant differences ( $p$-value of $<0.05$ ) by one-way analysis of variance and to calculate the correlation coefficient values for a correlation map. The correlation network map between metabolite that have a Pearson's correlation coefficient value higher than 0.5 and bioactivities were constructed with the Cytoscape software (https:/ / www.cytoscape.org/ (accessed on 13 July 2021)). The identification of tentative metabolites was carried out by matching the molecular weights and molecular composition, retention time, mass fragment 
patterns, and absorbance of ultraviolet (UV) data from the literature and our in-house library.

\subsection{Determination of Enzymatic Activities}

Enzymatic activity assays for $\alpha$-amylase, $\beta$-glucosidase, and $\alpha$-glucosidase were performed according to previous studies $[25,40,41]$. A $10 \mathrm{~g}$ quantity of each rice koji sample was extracted in $90 \mathrm{~mL}$ of water by shaking on an orbital shaker at $120 \mathrm{rpm}$ and $25^{\circ} \mathrm{C}$ for $1 \mathrm{~h}$. After filtering the samples, the supernatants were used to evaluate enzyme activities.

\subsection{Determination of Antioxidant Activities and Total Phenolic and Flavonoid Contents}

To determine the antioxidant activity of the rice koji samples, ABTS, DPPH, ferric reducing antioxidant power (FRAP), total phenolic contents (TPC), and total flavonoid contents (TFC) assays were conducted in triplicate.

The ABTS and FRAP assays were performed using the method described by Lee et al. [24]. In brief, the ABTS stock solution diluted with distilled water to achieve a final absorbance of $0.7 \pm 0.02$ at $750 \mathrm{~nm}(180 \mu \mathrm{L})$ was added to each sample extract $(20 \mu \mathrm{L})$ in a 96-well plate. The reaction was allowed to take place for $6 \mathrm{~min}$ in the dark at room temperature. The absorbance was measured at $750 \mathrm{~nm}$ using a spectrophotometer. For the FRAP assay, a mixture of $300 \mathrm{mM}$ acetate buffer (pH 3.6), $20 \mathrm{mM}$ iron (III) chloride, and $10 \mathrm{mM}$ 2,4,6-tripyridyl-S-triazine (TPTZ) solution in $40 \mathrm{mM} \mathrm{HCl}(10: 1: 1, v / v / v)$ was prepared. The sample $(10 \mu \mathrm{L})$ was mixed with $300 \mu \mathrm{L}$ of FRAP reagent and incubated at room temperature for $6 \mathrm{~min}$. The absorbance was measured at $570 \mathrm{~nm}$. The DPPH assay was carried out following the method adapted from Won et al. [42], where $180 \mu \mathrm{L}$ of the DPPH stock solution $(0.2 \mathrm{mM}$ in ethanol) was mixed with $20 \mu \mathrm{L}$ of the rice koji with two different fungal extracts in 96-well plates and allowed to react for $20 \mathrm{~min}$ at room temperature in the dark. The free radical absorbance by DPPH was measured at $515 \mathrm{~nm}$. The results of ABTS, FRAP, and DPPH are represented as the Trolox equivalent antioxidant capacity (TEAC) concentration (mM) per milligram of koji. The standard concentration curves ranged from $0.0078 \mathrm{mM}$ to $1 \mathrm{mM}$ TEAC.

For the TFC and TPC assays, a method used by Lee et al. [25] was followed. For the TFC assay, $20 \mu \mathrm{L}$ of each rice koji sample was mixed with $20 \mu \mathrm{L}$ of $1 \mathrm{~N} \mathrm{NaOH}$ and $180 \mu \mathrm{L}$ of $90 \%$ diethylene glycol in a 96-well plate. After incubation of the mixture for $60 \mathrm{~min}$ at room temperature, the absorbance was measured at $405 \mathrm{~nm}$. TFC is presented as the naringin equivalent (NE) concentration (mM) per milligram of koji. The standard concentration curve was linear between 0.0027 and $0.3445 \mathrm{mM}$ NE. For the analysis of the TPC assay, $20 \mu \mathrm{L}$ of each sample was incubated with $100 \mu \mathrm{L}$ of $0.2 \mathrm{~N}$ Folin-Ciocalteu reagent in 96-well plates at room temperature for $6 \mathrm{~min}$. Then, $80 \mu \mathrm{L}$ of $7.5 \%$ sodium carbonate $\left(\mathrm{Na}_{2} \mathrm{CO}_{3}\right)$ solution was added to the mixture and allowed to react for $60 \mathrm{~min}$ at room temperature. Finally, the absorbance was evaluated at $750 \mathrm{~nm}$. The results are indicated as gallic acid equivalent (GE) concentrations (mM) per milligram of koji in a standard concentration range of $0.0230-2.9391 \mathrm{mM} \mathrm{GE}$.

\subsection{Cell Cultures}

Primary human dermal fibroblasts (HDFs), a related culture medium, and DetachKit were purchased from PromoCell (Heidelberg, Germany). The HDFs were cultured in specific fibroblast medium (Fibroblast Growth Medium 2, PromoCell, Cat no. C-23020) abundant with Supplement Mix/Fibroblast Growth Medium 2 (PromoCell, Cat no. C39325 ) and $1 \%$ penicillin-streptomycin (PS) at $37^{\circ} \mathrm{C}$ in a $5 \% \mathrm{CO}_{2}$ incubator. When the cultured HDFs reached almost $80 \%$ confluence, they were sub-cultured or seeded into the proper wells for the different treatments and further assays.

\subsection{Real-Time Polymerase Chain Reaction}

To isolate and quantify the total RNA from the cell pellets, Trizol reagent was used, and the analysis was done using a spectrophotometer. The synthesis of cDNA was carried 
out in a total reaction volume of $20 \mu \mathrm{L}$; the reaction mixture consisted of $2 \mu \mathrm{g}$ of total RNA, oligo (dT), and reverse transcription premix under the following reaction conditions: $45{ }^{\circ} \mathrm{C}$ for $45 \mathrm{~min}$, followed by $95^{\circ} \mathrm{C}$ for $5 \mathrm{~min}$. RT-PCR was used for quantification of gene expression, and the results were subsequently analyzed using the StepOne PlusTM system software (Applied Biosystems, Foster City, CA, USA). RT-PCR amplifications were conducted using SYBR Green PCR Master Mix with premixed ROX (Applied Biosystems, Foster City, CA, USA) and primers (Bioneer, Daejeon, Korea) in an ABI 7300 instrument following the manufacturer's protocol. The reaction conditions were as follows: initiation at $95{ }^{\circ} \mathrm{C}$ for $10 \mathrm{~min}$, followed by cycling conditions of $95^{\circ} \mathrm{C}$ for $15 \mathrm{~s}, 60{ }^{\circ} \mathrm{C}$ for $30 \mathrm{~s}$, and $72{ }^{\circ} \mathrm{C}$ for $30 \mathrm{~s}$ for 40 cycles. $\beta$-actin was used as an internal control.

\section{Conclusions}

In conclusion, rice koji showed the production of different metabolites and bioactivities according to different Aspergillus species used. The higher levels of flavonoids and auroglaucin derivatives in RAC resulted in a higher antioxidant activity than in RAO. In addition, the synergistic effects of fatty acid and antioxidant compounds found in both the koji were associated with the RNA expression of the skin anti-aging factor. Auroglaucin derivatives and lysophospholipids found in RAC were also candidates that could be associated with RNA expression of skin anti-aging factors. Therefore, even though rice koji is fermented using members of the same genus (Aspergillus), there are significant differences in enzyme activities and metabolites for the different species, and they affect bioactivities such as antioxidant and anti-aging activities. Hence, this study provides comprehensive insight, as well as logic for a rational choice of inoculation microbes, with respect to metabolomics, to improve the quality of commercial production of koji.

Supplementary Materials: The following are available online at https:/ / www.mdpi.com/article/10 .3390/metabo11080524/s1, Figure S1: The PLS-DA score plot (A,B) and OPLS-DA score plot (C) for rice koji fermented with Aspergillus cristatus or A. oryzae were obtained from UHPLC-LTQ-OrbitrapMS/MS (A,C) and GC-TOF-MS (B)., Table S1: List of significantly distinct metabolites from rice koji with different Aspergillus spp. during fermentation identified by UHPLC-LTQ-Orbitrap-MS/MS, Table S2: List of significantly distinct metabolites from rice koji with different Aspergillus spp. during fermentation identified by GC-TOF-MS, Figure S2: Correlation map of bioactivities (skin cell effect and antioxidant activity) and rice koji fermented with Aspergillus cristatus or A. oryzae metabolites according to Pearson's correlation coefficient. Each square indicates Pearson's correlation coefficient values $(\mathrm{r})$.

Author Contributions: Conceptualization, C.L. and S.L.; methodology, H.L., S.L., S.K. (Seoyeon Kyung) and J.R.; validation, H.L., S.L. and S.K. (Seoyeon Kyung); formal analysis, H.L. and S.K. (Seoyeon Kyung); investigation, H.L. and S.L.; resources, J.R., S.K. (Seunghyun Kang) and M.P.; writing—original draft preparation, H.L.; writing—review and editing, H.L. and S.L.; visualization, H.L.; supervision, S.L. and C.L.; project administration, S.K. (Seunghyun Kang), M.P., and C.L.; funding acquisition, C.L. All authors have read and agreed to the published version of the manuscript.

Funding: This work was supported by Korea Institute of Planning and Evaluation for Technology in Food, Agriculture and Forestry(IPET) through Agricultural Microbiome R\&D Program(The Strategic Initiative for Microbiomes in Agriculture and Food), funded by Ministry of Agriculture, Food and Rural Affairs(MAFRA) (Grant number 918011-04-3-HD020). Additionally, this work was supported by Korea Institute of Planning and Evaluation for Technology in Food, Agriculture, Forestry(IPET) through High Value-added Food Technology Development Program, funded by Ministry of Agriculture, Food and Rural Affairs(MAFRA) (grant number 318027-04-3-HD030).

Institutional Review Board Statement: Not applicable.

Informed Consent Statement: Not applicable.

Data Availability Statement: The data presented in this study are available on request from the corresponding author. 
Acknowledgments: This research was supported by the Konkuk University Researcher Fund in 2020.

Conflicts of Interest: The authors declare no conflict of interest.

\section{References}

1. Sanlier, N.; Gokcen, B.B.; Sezgin, A.C. Health benefits of fermented foods. Crit. Rev. Food Sci. Nutr. 2019, 59, 506-527. [CrossRef]

2. Yu, K.-W.; Lee, S.-E.; Choi, H.-S.; Suh, H.J.; Ra, K.S.; Choi, J.W.; Hwang, J.-H. Optimization for rice koji preparation using Aspergillus oryzae CJCM-4 isolated from a Korean traditional meju. Food Sci. Biotechnol. 2012, 21, 129-135. [CrossRef]

3. Yang, Y.; Xia, Y.; Lin, X.; Wang, G.; Zhang, H.; Xiong, Z.; Yu, H.; Yu, J.; Ai, L. Improvement of flavor profiles in Chinese rice wine by creating fermenting yeast with superior ethanol tolerance and fermentation activity. Food Res. Int. 2018, 108, 83-92. [CrossRef] [PubMed]

4. Ichikawa, E.; Hirata, S.; Hata, Y.; Yazawa, H.; Tamura, H.; Kaneoke, M.; Iwashita, K.; Hirata, D. Effect of koji starter on metabolites in Japanese alcoholic beverage sake made from the sake rice Koshitanrei. Biosci. Biotechnol. Biochem. 2020, 84, 1714-1723. [CrossRef]

5. Phetpornpaisan, P.; Tippayawat, P.; Jay, M.; Sutthanut, K. A local Thai cultivar glutinous black rice bran: A source of functional compounds in immunomodulation, cell viability and collagen synthesis, and matrix metalloproteinase-2 and-9 inhibition. J. Funct. Foods 2014, 7, 650-661. [CrossRef]

6. Kim, A.J.; Choi, J.N.; Kim, J.; Kim, H.Y.; Park, S.B.; Yeo, S.H.; Choi, J.H.; Liu, K.H.; Lee, C.H. Metabolite profiling and bioactivity of rice koji fermented by Aspergillus strains. J. Microbiol. Biotechnol. 2012, 22, 100-106. [CrossRef] [PubMed]

7. Ames, B.N.; Shigenaga, M.K.; Hagen, T.M. Oxidants, antioxidants, and the degenerative diseases of aging. Proc. Natl. Acad. Sci. USA 1993, 90, 7915-7922. [CrossRef] [PubMed]

8. Valko, M.; Leibfritz, D.; Moncol, J.; Cronin, M.T.; Mazur, M.; Telser, J. Free radicals and antioxidants in normal physiological functions and human disease. Int. J. Biochem. Cell Biol. 2007, 39, 44-84. [CrossRef]

9. Uttara, B.; Singh, A.V.; Zamboni, P.; Mahajan, R.T. Oxidative stress and neurodegenerative diseases: A review of upstream and downstream antioxidant therapeutic options. Curr. Neuropharmacol. 2009, 7, 65-74. [CrossRef]

10. Yen, G.-C.; Chang, Y.-C.; Su, S.-W. Antioxidant activity and active compounds of rice koji fermented with Aspergillus candidus. Food Chem. 2003, 83, 49-54. [CrossRef]

11. Lee, D.E.; Lee, S.; Singh, D.; Jang, E.S.; Shin, H.W.; Moon, B.S.; Lee, C.H. Time-resolved comparative metabolomes for Koji fermentation with brown-, white-, and giant embryo-rice. Food Chem. 2017, 231, 258-266. [CrossRef] [PubMed]

12. Jarrar, M.; Behl, S.; Shaheen, N.; Fatima, A.; Nasab, R. Anti-Aging Effects of Retinol and Alpha Hydroxy Acid on Elastin Fibers of Artificially Photo-Aged Human Dermal Fibroblast Cell Lines. Int. J. Med Health Biomed. Pharm. Eng. 2015, 7, 328.

13. Bolla, S.R.; Al-Subaie, A.M.; Al-Jindan, R.Y.; Balakrishna, J.P.; Ravi, P.K.; Veeraraghavan, V.P.; Pillai, A.A.; Gollapalli, S.S.R.; Joseph, J.P.; Surapaneni, K.M. In vitro wound healing potency of methanolic leaf extract of Aristolochia saccata is possibly mediated by its stimulatory effect on collagen-1 expression. Heliyon 2019, 5, e01648. [CrossRef] [PubMed]

14. Meinke, M.C.; Nowbary, C.K.; Schanzer, S.; Vollert, H.; Lademann, J.; Darvin, M.E. Influences of Orally Taken Carotenoid-Rich Curly Kale Extract on Collagen I/Elastin Index of the Skin. Nutrients 2017, 9, 775. [CrossRef] [PubMed]

15. Majeed, M.; Bhat, B.; Anand, S.; Sivakumar, A.; Paliwal, P.; Geetha, K.G. Inhibition of UV-induced ROS and collagen damage by Phyllanthus emblica extract in normal human dermal fibroblasts. J. Cosmet. Sci. 2011, 62, 49-56. [PubMed]

16. Masuda, M.; Murata, K.; Naruto, S.; Uwaya, A.; Isami, F.; Matsuda, H. Matrix metalloproteinase-1 inhibitory activities of Morinda citrifolia seed extract and its constituents in UVA-irradiated human dermal fibroblasts. Biol. Pharm. Bull. 2012, 35, 210-215. [CrossRef] [PubMed]

17. Seo, Y.-K.; Jung, S.-H.; Song, K.-Y.; Park, J.-K.; Park, C.-S. Anti-photoaging effect of fermented rice bran extract on UV-induced normal skin fibroblasts. Eur. Food Res. Technol. 2010, 231, 163-169. [CrossRef]

18. Goufo, P.; Trindade, H. Rice antioxidants: Phenolic acids, flavonoids, anthocyanins, proanthocyanidins, tocopherols, tocotrienols, $\gamma$-oryzanol, and phytic acid. Food Sci. Nutr. 2014, 2, 75-104. [CrossRef]

19. Bechman, A.; Phillips, R.D.; Chen, J. Changes in selected physical property and enzyme activity of rice and barley koji during fermentation and storage. J. Food. Sci. 2012, 77, M318-M322. [CrossRef]

20. Kang, D.; Su, M.; Duan, Y.; Huang, Y. Eurotium cristatum, a potential probiotic fungus from Fuzhuan brick tea, alleviated obesity in mice by modulating gut microbiota. Food Funct. 2019, 10, 5032-5045. [CrossRef]

21. Zhao, P.; Alam, M.B.; Lee, S.H. Protection of UVB-Induced Photoaging by Fuzhuan-Brick Tea Aqueous Extract via MAPKs/Nrf2Mediated Down-Regulation of MMP-1. Nutrients 2018, 11, 60. [CrossRef] [PubMed]

22. Hur, S.J.; Lee, S.Y.; Kim, Y.C.; Choi, I.; Kim, G.B. Effect of fermentation on the antioxidant activity in plant-based foods. Food Chem. 2014, 160, 346-356. [CrossRef] [PubMed]

23. Zhou, S.-D.; Xu, X.; Lin, Y.-F.; Xia, H.-Y.; Huang, L.; Dong, M.-S. On-line screening and identification of free radical scavenging compounds in Angelica dahurica fermented with Eurotium cristatum using an HPLC-PDA-Triple-TOF-MS/MS-ABTS system. Food Chem. 2019, 272, 670-678. [CrossRef] [PubMed]

24. Lee, S.; Lee, D.E.; Singh, D.; Lee, C.H. Metabolomics Reveal Optimal Grain Preprocessing (Milling) toward Rice Koji Fermentation. J. Agric. Food Chem. 2018, 66, 2694-2703. [CrossRef] 
25. Lee da, E.; Lee, S.; Jang, E.S.; Shin, H.W.; Moon, B.S.; Lee, C.H. Metabolomic Profiles of Aspergillus oryzae and Bacillus amyloliquefaciens During Rice Koji Fermentation. Molecules 2016, 21, 773. [CrossRef]

26. Friedman, M. Rice brans, rice bran oils, and rice hulls: Composition, food and industrial uses, and bioactivities in humans, animals, and cells. J. Agric. Food Chem. 2013, 61, 10626-10641. [CrossRef]

27. Rao, R.S.P.; Muralikrishna, G. Water soluble feruloyl arabinoxylans from rice and ragi: Changes upon malting and their consequence on antioxidant activity. Phytochemistry 2006, 67, 91-99. [CrossRef]

28. Kim, A.J.; Choi, J.N.; Kim, J.; Yeo, S.H.; Choi, J.H.; Lee, C.H. Metabolomics-based optimal koji fermentation for tyrosinase inhibition supplemented with Astragalus radix. Biosci. Biotechnol. Biochem. 2012, 76, 863-869. [CrossRef]

29. Walter, M.; Marchesan, E. Phenolic compounds and antioxidant activity of rice. Braz. Arch. Biol. Technol. 2011, 54, 371-377. [CrossRef]

30. Xiao, J. Dietary flavonoid aglycones and their glycosides: Which show better biological significance? Crit. Rev. Food Sci. Nutr. 2017, 57, 1874-1905. [CrossRef]

31. Chen, Y.; Wang, Y.; Chen, J.; Tang, H.; Wang, C.; Li, Z.; Xiao, Y. Bioprocessing of soybeans (Glycine max L.) by solid-state fermentation with Eurotium cristatum YL-1 improves total phenolic content, isoflavone aglycones, and antioxidant activity. RSC Adv. 2020, 10, 16928-16941. [CrossRef]

32. Miyake, Y.; Mochizuki, M.; Ito, C.; Itoigawa, M.; Osawa, T. Peroxynitrite scavengers produced by filamentous fungus used in the katsuobushi manufacturing process. Food Sci. Technol. Res. 2010, 16, 493-498. [CrossRef]

33. Masaki, H. Role of antioxidants in the skin: Anti-aging effects. J. Dermatol. Sci. 2010, 58, 85-90. [CrossRef] [PubMed]

34. Lin, T.-K.; Zhong, L.; Santiago, J.L. Anti-inflammatory and skin barrier repair effects of topical application of some plant oils. Int. J. Mol. Sci. 2018, 19, 70. [CrossRef] [PubMed]

35. Chen, A.J.; Hubka, V.; Frisvad, J.C.; Visagie, C.M.; Houbraken, J.; Meijer, M.; Varga, J.; Demirel, R.; Jurjevic, Z.; Kubatova, A.; et al. Polyphasic taxonomy of Aspergillus section Aspergillus (formerly Eurotium), and its occurrence in indoor environments and food. Stud. Mycol. 2017, 88, 37-135. [CrossRef]

36. Yahagi, S.; Koike, M.; Okano, Y.; Masaki, H. Lysophospholipids improve skin moisturization by modulating of calcium-dependent cell differentiation pathway. Int. J. Cosmet. Sci. 2011, 33, 251-256. [CrossRef] [PubMed]

37. Flynn, T.C.; Petros, J.; Clark, R.E.; Viehman, G.E. Dry skin and moisturizers. Clin. Dermatol. 2001, 19, 387-392. [CrossRef]

38. Yokota, M.; Shimizu, K.; Kyotani, D.; Yahagi, S.; Hashimoto, S.; Masaki, H. The possible involvement of skin dryness on alterations of the dermal matrix. Exp. Dermatol. 2014, 23, 27-31. [CrossRef] [PubMed]

39. Kwon, M.C.; Kim, Y.X.; Lee, S.; Jung, E.S.; Singh, D.; Sung, J.; Lee, C.H. Comparative Metabolomics Unravel the Effect of Magnesium Oversupply on Tomato Fruit Quality and Associated Plant Metabolism. Metabolites 2019, 9, 231. [CrossRef] [PubMed]

40. Rudick, M.J.; Fitzgerald, Z.E.; Rudick, V.L. Intra-and extracellular forms of $\alpha$-glucosidase from Aspergillus niger. Arch. Biochem. Biophys. 1979, 193, 509-520. [CrossRef]

41. Del Moral, S.; Barradas-Dermitz, D.; Aguilar-Uscanga, M. Production and biochemical characterization of $\alpha$-glucosidase from Aspergillus niger ITV-01 isolated from sugar cane bagasse. 3 Biotech. 2018, 8, 1-9. [CrossRef] [PubMed]

42. Won, J.Y.; Son, S.Y.; Lee, S.; Singh, D.; Lee, S.; Lee, J.S.; Lee, C.H. Strategy for Screening of Antioxidant Compounds from Two Ulmaceae Species Based on Liquid Chromatography-Mass Spectrometry. Molecules 2018, 23, 1830. [CrossRef] [PubMed] 